\title{
Self-assembly cloning: a rapid construction method for recombinant molecules from multiple fragments
}

\section{Protocol For: \\ Self-assembly cloning: a rapid construction method for recombinant molecules from multiple fragments}

Akira Matsumoto ${ }^{1}$ and Taichi Q. Itoh 2,3

${ }^{1}$ Department of Biology, Juntendo University School of Medicine, Inzai, Chiba, Japan, ${ }^{2}$ Graduate School of Systems Life Sciences, Kyushu University, Hakozaki, Fukuoka, Japan, and ${ }^{3}$ Research

Fellow of the Japan Society for the Promotion of Science, Japan

BioTechniques Protocol Guide, January 2017; doi 10.2144/000114507

See full protocol online: https://benchtalk.biotechniques.com/users/8521-biotechniques-editors/posts/11342-protocol-for-self-assembly-cloninga-rapid-construction-method-for-recombinant-molecules-from-multiple-fragments

Protocol Summary: Enzyme-free cloning (EFC) can rapidly produce an in-frame fusion gene with multiple fragments. To practically apply EFC, we investigated the extent and sequence of complementary staggered overhangs necessary to direct self-assembly of multiple fragments as well as a size limitation of the constructed DNA molecule. Six-base pair overhangs with 50\% GC content were sufficient to direct self-assembly. A functional plasmid that exceeded $10 \mathrm{~kb}$, which includes an in-frame fusion domain, was efficiently constructed from four PCR fragments in one step by our improved method.

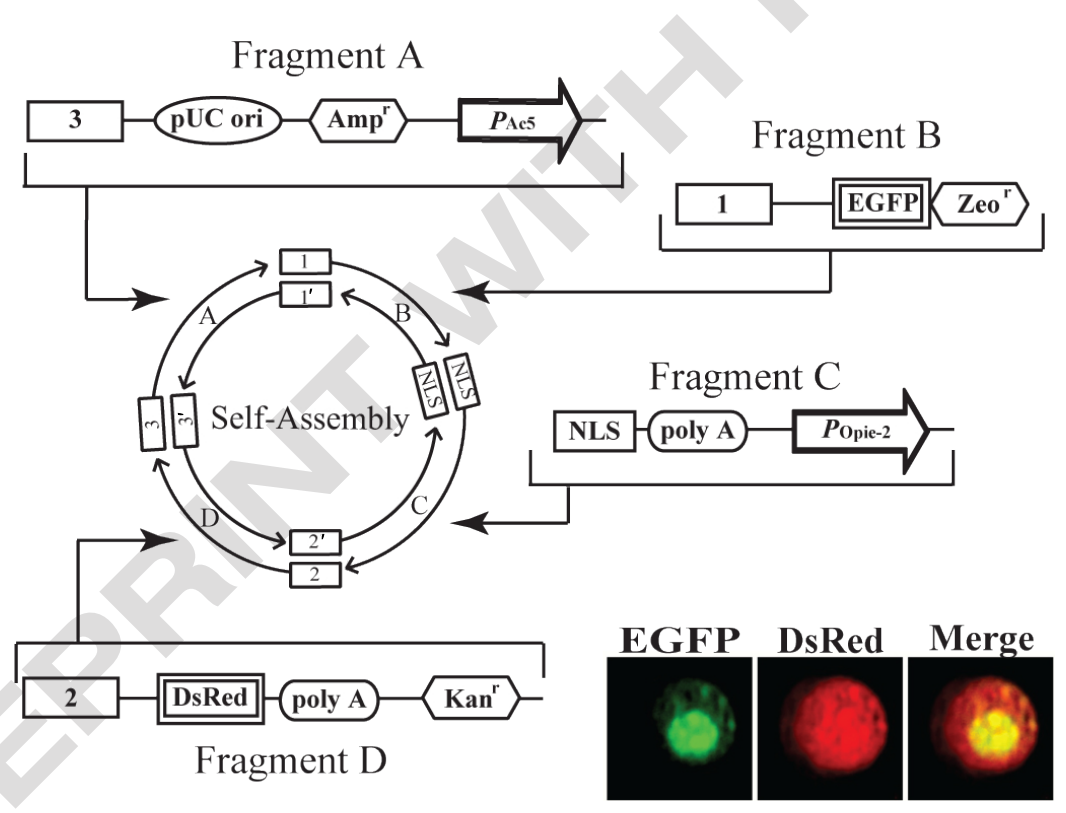

Protocol Schematic: A multifunctional plasmid created by SA cloning that expresses reporter proteins in different subcellular compartments in insect cells. The plasmid, designed to self-assemble with four fragments, has several functional units: a replication origin (represented as an oval); three antibiotic resistance genes for amplicillin, zeocin, and kanamycin (a hexagon); two genes encoding the fluorescent reporter proteins enhanced GFP (EGFP) and DsRed (a double box) are driven by PAc5 and POpie-2 promoters (an arrow) and terminated by the polyA signal sequences (a roundedged rectangle), respectively; an NLS (box) fused in-frame to EGFP-Zeo, in which the NLS sequence was also used as a complementary overhang to self-assemble between fragments $B$ and $C$. Only the sense strand of each fragment with the $5^{\prime}$ overhang region (a numbered box) is illustrated around the designed plasmid. The signal of EGFP is shown as green in nucleus (left panel of insets) and red in cytoplasm (middle). The right panel represents the merge of EGFP and DsRed signals. 